\section{ENTWICKLUNG DES BIP}

Veränderungen der Größe von Volkswirtschaften werden in der Regel anhand von Veränderungen des BIP-Volumens (oft als reales BIP bezeichnet) gemessen. „Real“ bedeutet, dass inflationsbedingte Veränderungen des BIP herausgerechnet werden. Dadurch erhält man eine Messgröße für die Veränderung des Produktionsvolumens einer Volkswirtschaft.

\section{Definition}

Für die Umrechnung nominaler BIP-Werte in reale Werte sind eine Reihe implizit oder direkt erfasster detaillierter Preisindizes erforderlich. Mit Hilfe dieser Preisindizes können aus dem nominalen Wert der Transaktionen die entsprechenden Volumenänderungen ermittelt werden. Seit Einführung des Systems der Volkswirtschaftlichen Gesamtrechnungen von 1993 (SNA 1993) wird empfohlen, dass die Gewichtungen für die Zeiträume repräsentativ sein sollten, für die die Wachstumsraten berechnet werden. Das bedeutet, dass jedes Jahr neue Gewichtungen eingeführt werden sollten, aus denen sich verkettete (Volumen-)Indizes ergeben.

\section{Vergleichbarkeit}

Alle Länder erfassen Daten nach dem System der Volkswirtschaftlichen Gesamtrechnungen von 1993 (SNA 1993), mit Ausnahme Australiens, wo die Daten nach dem neuen SNA 2008 erfasst werden. Dabei ist jedoch zu beachten, dass die Unterschiede zwischen SNA 2008 und SNA 1993 keinen wesentlichen Einfluss auf die Vergleichbarkeit der hier dargestellten Indikatoren haben, weshalb die Daten der einzelnen Länder gut miteinander vergleichbar sind. Es gibt aber im Allgemeinen

\section{Überblick}

Die jahresdurchschnittliche Zuwachsrate des BIP-Volumens für den OECD-Raum insgesamt belief sich in den drei Jahren bis 2011 auf 0,3\%. Zurückzuführen ist dies in erster Linie auf den drastischen Einbruch um 3,8\% im Jahr 2009, als die jüngste Wirtschaftskrise ihren Höhepunkt erreichte. Das ist im Verhältnis zu den deutlich höheren BIP-Wachstumsraten in Indien, Indonesien und China zu sehen, wo die jahresdurchschnittlichen Zuwachsraten im genannten Zeitraum überall mindestens $5 \%$ betrugen. Griechenland, Island, Slowenien und Irland, deren Wirtschaft um 2-5\% schrumpfte, verzeichneten zwischen 2008 und 2011 die niedrigsten jahresdurchschnittlichen BIPWachstumsraten. Insgesamt wiesen in diesem Zeitraum 16 OECD-Länder eine negative jahresdurchschnittliche Zuwachsrate aus. Das zeigt, dass es diesen Ländern noch immer nicht gelungen ist, die krisenbedingten Einbußen bei der Wirtschaftstätigkeit auszugleichen und wieder ein BIP auf Vorkrisenniveau zu erreichen.

Für das Jahr 2011 allein lag die Wachstumsrate im OECDRaum bei $1,8 \%$. Chile (6,0\%), Estland $(7,6 \%)$ und die Türkei $(8,5 \%)$ verzeichneten die höchsten Wachstumsraten. In Griechenland dagegen schrumpfte die wirtschaftliche Aktivität um 6,9\%. Auch Portugal (minus 1,6\%), Japan (minus 0,7\%) und Slowenien (minus 0,2\%) wiesen 2011 ein Negativwachstum aus. in den einzelnen Ländern Unterschiede bei den Berechnungsmodalitäten der BIP-Volumenschätzungen, insbesondere hinsichtlich vom Staat erbrachter Dienstleistungen, etwa im Gesundheits- und Bildungswesen.

Mit Ausnahme Mexikos leiten alle OECD-Länder ihre jährlichen Schätzungen des realen BIP von jährlich verketteten Volumenindizes ab (d.h. die Gewichtungen werden jedes Jahr aktualisiert). Mexiko passt, ebenso wie viele Nicht-OECDLänder, die Gewichtungen weniger häufig an.

\section{Reales BIP-Wachstum}

Jährliche Veränderung in Prozent

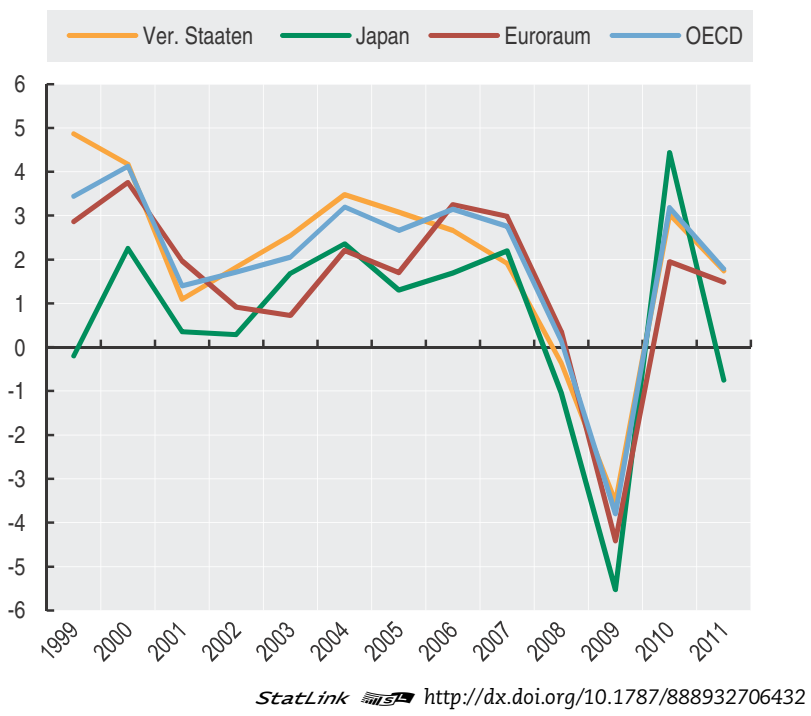

Quelle

- OECD (2012), National Accounts of OECD Countries, OECD Publishing.

- Für Nichtmitgliedsländer: Nationale Quellen.

Weitere Informationen

Analysen

- OECD (2012), OECD-Wirtschaftsausblick, OECD Publishing.

- OECD (2012), Economic Policy Reforms, OECD Publishing.

- OECD (2012), OECD Journal: Economic Studies, OECD Publishing.

Statistiken

- OECD (2011), National Accounts at a Glance, OECD Publishing.

Online-Datenbanken

- OECD National Accounts Statistics.

- OECD Economic Outlook: Statistics and Projections.

Websites

- OECD Economic Outlook - Sources and Methods, www.oecd.org/eco/sources-and-methods. 
Reales BIP-Wachstum

Jährliche Veränderung in Prozent

\begin{tabular}{|c|c|c|c|c|c|c|c|c|c|c|c|c|c|}
\hline & 1999 & 2000 & 2001 & 2002 & 2003 & 2004 & 2005 & 2006 & 2007 & 2008 & 2009 & 2010 & 2011 \\
\hline Australien & 3.8 & 1.9 & 3.9 & 3.2 & 4.1 & 3.2 & 3.0 & 3.8 & 3.8 & 1.4 & 2.3 & 2.1 & 1.8 \\
\hline Belgien & 3.5 & 3.7 & 0.8 & 1.4 & 0.8 & 3.3 & 1.8 & 2.7 & 2.9 & 1.0 & -2.8 & 2.2 & 1.9 \\
\hline Chile & -0.7 & 4.5 & 3.3 & 2.2 & 4.0 & 7.0 & 6.2 & 5.7 & 5.2 & 3.3 & -1.0 & 6.1 & 6.0 \\
\hline Dänemark & 2.6 & 3.5 & 0.7 & 0.5 & 0.4 & 2.3 & 2.4 & 3.4 & 1.6 & -0.8 & -5.8 & 1.3 & 0.8 \\
\hline Deutschland & 1.9 & 3.1 & 1.5 & 0.0 & -0.4 & 1.2 & 0.7 & 3.7 & 3.3 & 1.1 & -5.1 & 3.7 & 3.0 \\
\hline Estland & -0.3 & 9.7 & 6.3 & 6.6 & 7.8 & 6.3 & 8.9 & 10.1 & 7.5 & -3.7 & -14.3 & 2.3 & 7.6 \\
\hline Finnland & 3.9 & 5.3 & 2.3 & 1.8 & 2.0 & 4.1 & 2.9 & 4.4 & 5.3 & 0.3 & -8.5 & 3.3 & 2.7 \\
\hline Frankreich & 3.3 & 3.7 & 1.8 & 0.9 & 0.9 & 2.5 & 1.8 & 2.5 & 2.3 & -0.1 & -3.1 & 1.7 & 1.7 \\
\hline Griechenland & 3.4 & 4.5 & 4.2 & 3.4 & 5.9 & 4.4 & 2.3 & 5.5 & 3.0 & -0.2 & -3.2 & -3.5 & -6.9 \\
\hline Irland & 9.9 & 9.3 & 4.8 & 5.9 & 4.2 & 4.5 & 5.3 & 5.3 & 5.2 & -3.0 & -7.0 & -0.4 & 0.7 \\
\hline Island & 4.1 & 4.3 & 3.9 & 0.1 & 2.4 & 7.8 & 7.2 & 4.7 & 6.0 & 1.3 & -6.8 & -4.0 & 3.1 \\
\hline Israel & 3.4 & 9.3 & -0.2 & -0.6 & 1.5 & 4.8 & 4.9 & 5.6 & 5.5 & 4.0 & 0.8 & 4.8 & 4.8 \\
\hline Italien & 1.5 & 3.7 & 1.9 & 0.5 & 0.0 & 1.7 & 0.9 & 2.2 & 1.7 & -1.2 & -5.5 & 1.8 & 0.4 \\
\hline Japan & -0.2 & 2.3 & 0.4 & 0.3 & 1.7 & 2.4 & 1.3 & 1.7 & 2.2 & -1.0 & -5.5 & 4.4 & -0.7 \\
\hline Kanada & 5.5 & 5.2 & 1.8 & 2.9 & 1.9 & 3.1 & 3.0 & 2.8 & 2.2 & 0.7 & -2.8 & 3.2 & 2.5 \\
\hline Korea & 10.7 & 8.8 & 4.0 & 7.2 & 2.8 & 4.6 & 4.0 & 5.2 & 5.1 & 2.3 & 0.3 & 6.3 & 3.6 \\
\hline Luxemburg & 8.4 & 8.4 & 2.5 & 4.1 & 1.5 & 4.4 & 5.4 & 5.0 & 6.6 & 0.8 & -5.3 & 2.7 & 1.6 \\
\hline Mexiko & 3.8 & 6.6 & 0.0 & 0.8 & 1.4 & 4.1 & 3.3 & 5.1 & 3.4 & 1.2 & -6.3 & 5.6 & 3.9 \\
\hline Neuseeland & 5.2 & 2.5 & 3.5 & 4.9 & 3.9 & 3.6 & 3.2 & 2.2 & 2.9 & -1.1 & 0.8 & 1.2 & 0.3 \\
\hline Niederlande & 4.7 & 3.9 & 1.9 & 0.1 & 0.3 & 2.2 & 2.0 & 3.4 & 3.9 & 1.8 & -3.5 & 1.7 & 1.2 \\
\hline Norwegen & 2.0 & 3.3 & 2.0 & 1.5 & 1.0 & 4.0 & 2.6 & 2.5 & 2.7 & 0.0 & -1.7 & 0.7 & 1.4 \\
\hline Österreich & 3.5 & 3.7 & 0.9 & 1.7 & 0.9 & 2.6 & 2.4 & 3.7 & 3.7 & 1.4 & -3.8 & 2.1 & 2.7 \\
\hline Polen & 4.5 & 4.3 & 1.2 & 1.4 & 3.9 & 5.3 & 3.6 & 6.2 & 6.8 & 5.1 & 1.6 & 3.9 & 4.3 \\
\hline Portugal & 4.1 & 3.9 & 2.0 & 0.8 & -0.9 & 1.6 & 0.8 & 1.4 & 2.4 & 0.0 & -2.9 & 1.4 & -1.6 \\
\hline Schweden & 4.7 & 4.5 & 1.3 & 2.5 & 2.3 & 4.2 & 3.2 & 4.3 & 3.3 & -0.6 & -5.0 & 6.2 & 3.9 \\
\hline Schweiz & 1.4 & 3.7 & 1.2 & 0.2 & 0.0 & 2.4 & 2.7 & 3.8 & 3.8 & 2.2 & -1.9 & 3.0 & 2.1 \\
\hline Slowak. Rep. & 0.0 & 1.4 & 3.5 & 4.6 & 4.8 & 5.1 & 6.7 & 8.3 & 10.5 & 5.8 & -4.9 & 4.2 & 3.3 \\
\hline Slowenien & 5.3 & 4.3 & 2.9 & 3.8 & 2.9 & 4.4 & 4.0 & 5.8 & 6.9 & 3.6 & -8.0 & 1.4 & -0.2 \\
\hline Spanien & 4.7 & 5.0 & 3.7 & 2.7 & 3.1 & 3.3 & 3.6 & 4.1 & 3.5 & 0.9 & -3.7 & -0.1 & 0.7 \\
\hline Tschech. Rep. & 1.7 & 4.2 & 3.1 & 2.1 & 3.8 & 4.7 & 6.8 & 7.0 & 5.7 & 3.1 & -4.7 & 2.7 & 1.7 \\
\hline Türkei & -3.4 & 6.8 & -5.7 & 6.2 & 5.3 & 9.4 & 8.4 & 6.9 & 4.7 & 0.7 & -4.8 & 9.2 & 8.5 \\
\hline Ungarn & 3.2 & 4.2 & 3.7 & 4.5 & 3.9 & 4.8 & 4.0 & 3.9 & 0.1 & 0.9 & -6.8 & 1.3 & 1.6 \\
\hline Ver. Königreich & 3.2 & 4.2 & 2.9 & 2.4 & 3.8 & 2.9 & 2.8 & 2.6 & 3.6 & -1.0 & -4.0 & 1.8 & 0.8 \\
\hline Ver. Staaten & 4.9 & 4.2 & 1.1 & 1.8 & 2.6 & 3.5 & 3.1 & 2.7 & 1.9 & -0.4 & -3.5 & 3.0 & 1.7 \\
\hline Euroraum & 2.9 & 3.8 & 2.0 & 0.9 & 0.7 & 2.2 & 1.7 & 3.3 & 3.0 & 0.4 & -4.4 & 2.0 & 1.5 \\
\hline EU27 & 3.0 & 3.9 & 2.2 & 1.3 & 1.4 & 2.5 & 2.0 & 3.3 & 3.2 & 0.3 & -4.4 & 2.1 & 1.5 \\
\hline OECD & 3.4 & 4.1 & 1.4 & 1.7 & 2.1 & 3.2 & 2.7 & 3.2 & 2.8 & 0.1 & -3.8 & 3.2 & 1.8 \\
\hline Brasilien & 0.3 & 4.3 & 1.3 & 2.7 & 1.1 & 5.7 & 3.2 & 4.0 & 6.1 & 5.2 & -0.6 & 7.5 &. \\
\hline China & 7.6 & 8.4 & 8.3 & 9.1 & 10.0 & 10.1 & 11.3 & 12.7 & 14.2 & 9.6 & 9.2 & 10.3 & .. \\
\hline Indien & 3.3 & 4.4 & 3.9 & 4.6 & 6.9 & 8.1 & 9.2 & 9.7 & 9.9 & 6.2 & 6.8 & 10.4 & .. \\
\hline Indonesien & 0.8 & 5.4 & 3.6 & 4.5 & 4.8 & 5.0 & 5.7 & 5.5 & 6.3 & 6.0 & 4.6 & 6.1 & .. \\
\hline Russ. Föderation & 6.4 & 10.0 & 5.1 & 4.7 & 7.3 & 7.2 & 6.4 & 8.2 & 8.5 & 5.2 & -7.8 & 4.3 & 4.3 \\
\hline Südafrika & 2.4 & 4.2 & 2.7 & 3.7 & 2.9 & 4.6 & 5.3 & 5.6 & 5.6 & 3.6 & -1.7 & 2.8 & .. \\
\hline
\end{tabular}

Reales BIP-Wachstum

Durchschnittliche jährliche Veränderung in Prozent

Dreijahresdurchschnitt am Ende des Zeitraums (2008-2010) • Dreijahresdurchschnitt zu Beginn des Zeitraums (1999-2001)

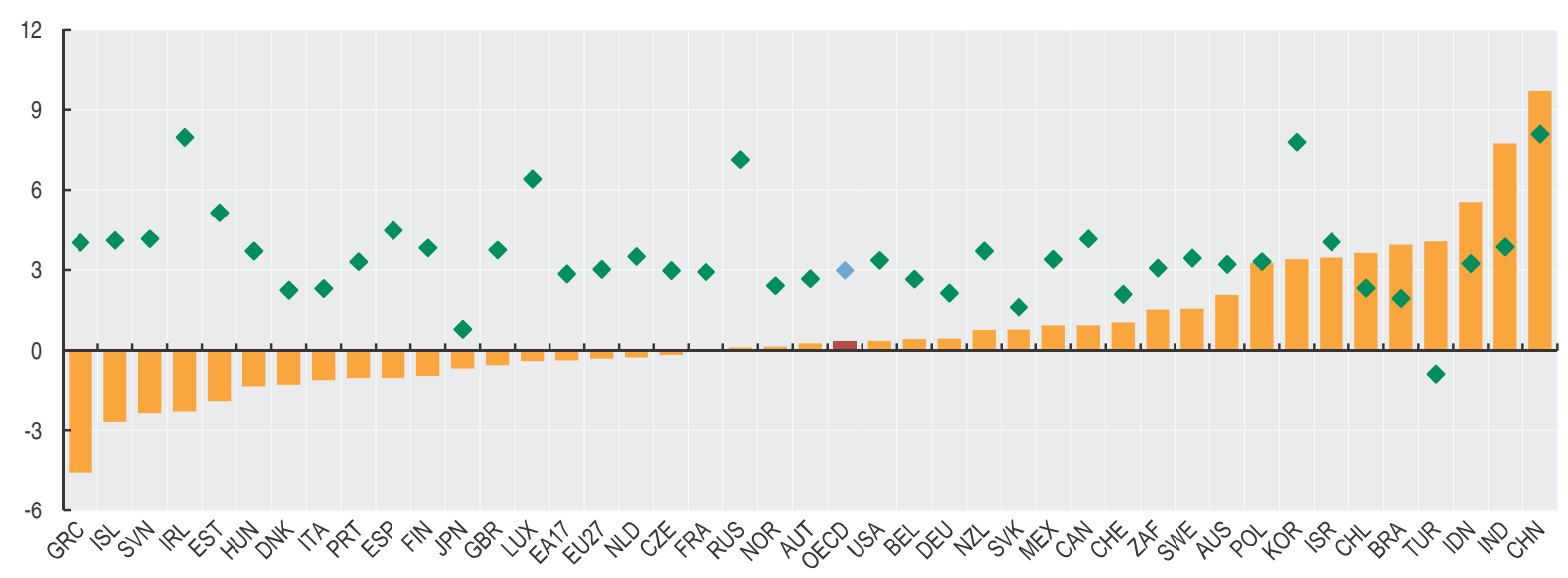




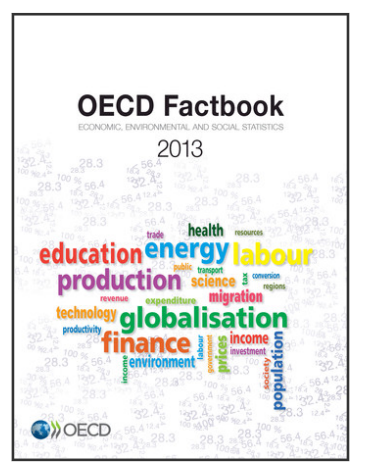

\section{From: \\ OECD Factbook 2013 \\ Economic, Environmental and Social Statistics}

Access the complete publication at:

https://doi.org/10.1787/factbook-2013-en

Please cite this chapter as:

OECD (2013), "Entwicklung des BIP”, in OECD Factbook 2013: Economic, Environmental and Social Statistics, OECD Publishing, Paris.

DOI: https://doi.org/10.1787/factbook-2013-11-de

Das vorliegende Dokument wird unter der Verantwortung des Generalsekretärs der OECD veröffentlicht. Die darin zum Ausdruck gebrachten Meinungen und Argumente spiegeln nicht zwangsläufig die offizielle Einstellung der OECDMitgliedstaaten wider.

This document and any map included herein are without prejudice to the status of or sovereignty over any territory, to the delimitation of international frontiers and boundaries and to the name of any territory, city or area.

You can copy, download or print OECD content for your own use, and you can include excerpts from OECD publications, databases and multimedia products in your own documents, presentations, blogs, websites and teaching materials, provided that suitable acknowledgment of OECD as source and copyright owner is given. All requests for public or commercial use and translation rights should be submitted to rights@oecd.org. Requests for permission to photocopy portions of this material for public or commercial use shall be addressed directly to the Copyright Clearance Center (CCC) at info@copyright.com or the Centre français d'exploitation du droit de copie (CFC) at contact@cfcopies.com. 